\title{
Assessment of MANET Broadcast Schemes in the Application Context of Multiplayer Video Games
}

\author{
Ahmad Sardouk and Sidi Mohammed \\ Senouci \\ Orange Labs - CORE/M2I \\ 2, Avenue Pierre Marzin \\ 22307 Lannion, France \\ ahmad.sardouk@orange- \\ ftgroup.com,sidimohammed.senouci@orange- \\ ftgroup.com \\ Nadjib Achir and Khaled Boussetta \\ L2TI - Institut Galilee - University Paris 13 \\ 99 Avenue J-B Clement \\ 93430 Villetaneuse, France \\ nadjib.achir@|2ti.univ- \\ paris13.fr,khaled.boussetta@|2ti.univ- \\ paris13.fr
}

\begin{abstract}
Taking into consideration the popularity of multiplayer games and the tremendous success of wireless networks, we strongly believe that future game engines will benefit from the integration of MANET (Mobile Ad hoc NETworks) technology [1]. Indeed, in addition to the social interests of playing with close located persons, MANET technology will allow players, like a group of kids in a playground, to easily improvise a LAN multiplayer party without any need for an existing wireless network infrastructure. In order to make such scenario a common based reality, several issues still have to be addressed. Among which is the MANET's broadcast problem. In fact, broadcast is a fundamental network service for multiplayer LAN mode. But up to now, the most used scheme for broadcasting in MANET relies on flooding. Unfortunately, this simple scheme leads to high redundancy and collisions. In the context of a multiplayer party supported by mobile terminals, this broadcast storm problem [10], could result into a dramatic reduction of the network energy-based lifetime and to a high variability of the transmission delay. The latter one is know to be a crucial QoS parameter which can significantly affect the game play. In this paper, we evaluate the performance of different MANET broadcast schemes in the context of multiplayer video games. We analyze the capacity of simple flooding, distance based, probability based and self pruning broadcast schemes to satisfy the QoS constraints required by multiplayer games like FPS. Assessment simulations are conducted for both Client/Server and P2P network game architectures.
\end{abstract}

\section{Categories and Subject Descriptors}

C.2.1 [Computer Systems Organization]: ComputerCommunication Networks - Network Architecture and Design, Network Protocols, Distributed Systems.; C.4.1 [Computer

Permission to make digital or hard copies of all or part of this work for personal or classroom use is granted without fee provided that copies are not made or distributed for profit or commercial advantage and that copies bear this notice and the full citation on the first page. To copy otherwise, to republish, to post on servers or to redistribute to lists, requires prior specific permission from the authors.

NetGames '07, September 19-20, 2007, Melbourne, Australia
Systems Organization]: Performance of Systems - Performance attributes Reliability, availability, and serviceability.

\section{General Terms}

Algorithms, Performance, Reliability.

\section{Keywords}

Multiplayer video games, ad hoc networks, broadcast.

\section{INTRODUCTION}

Up to now, the multiplayer mode is generally supported by wired communication infrastructures. Wireless links, based on cellular technology (e.g. UMTS) or WiFi, could be used as well but only as a last link in the transmission path. This is typically the case with laptops, PDAs or recent consoles such as Nintendo Wii, Nintendo DS, Sony's Portable Playstation (PSP) and Microsoft Xbox, all whether offering a build-in WiFi interface or could be equipped by a USB WiFi connector. Thus, in this last generation of consoles LAN, multiplayer mode based on $\mathrm{WiFi}$ is now available. However, only one single hop is supported. In other words, in order to play together, all players must be located within the same radio coverage. Multi-hop radio communications using a MANET (Mobile Ad-hoc NETworks) [1] technology is not supported yet by the actual generation of consoles.

MANETs are an ultimate generalization of the wireless networks. Indeed, ad-hoc networks technology allows spontaneous creation of data networks by exploiting the capacity of the mobile terminals to communicate directly between them without the need of a centralized infrastructure. Terminals forming the ad-hoc network should play the role of routers in order to ensure the gradual transfer of data packets. Moreover, the network management tasks (autoconfiguration, security, etc.) are carried out by the terminals themselves in an entirely distributed way.

Taking into consideration the popularity of LAN multiplayer games and the tremendous success of wireless networks, we claim that future game engines will benefit from supporting MANET mode. In addition to the social and cultural interest of playing with close located persons, MANET technology could offer the mobility comfort for a much higher number of potential players than that actually supported by last generation of commercialized consoles. Other advantages of MANET technology for LAN multiparty gaming 
are the facility of deployment, the autonomic reconfiguration, the self-healing and the possibility of capacity addition without preliminary planning. All these elements make us strongly believe on the suitability of integration of MANET technology in future games terminals (e.g. portable consoles). A scenario, like a group of kids, of varying size, equipped with portable consoles supporting MANET services and wishing to play a WLAN party in a playground (without necessitating any particular fixed network infrastructure) will be possible.

In order to make such scenario a common based reality in the near future, several issues still have to be addressed by the research community. Several difficulties come from the inherent constraints that characterize MANET technology. In particular, the mobility of the core network, the energy limitation and the variability of shared resources. All these problems lead to a variable connectivity and potentially, to a high fluctuation in the provided Quality of Service (QoS). On the other hand, multiparty games are very sensitive to resource availability. They are very demanding on CPU and thus, high consuming on energy. In addition, the game play could experience a significant degradation caused by delays variability, which is typically the case in MANET, due to the mobility of users and to the probabilistic MAC layer nature. In particular, a specific MANET aspect which can impact on the game play is the broadcast issue.

Indeed, in many LAN games, a large amount of messages generated by the entities evolved in the party are exchanged through MAC broadcast messages. A simplest and commonly used scheme for broadcast in MANET is based on a simple flooding mechanism. Unfortunately, when several nodes are involved in the broadcast process and when all of them compete to transmit, the simple flooding scheme could raise a well known problem, referred as the broadcast storm problem [10].

In the literature, several mechanisms have been proposed in order to optimize the MANET's broadcast in term of reliability, efficiency, energy consumption and ability to satisfy the application's QoS constraints. Assessment of the proposed schemes was conducted for several applications like video or audio streaming, file sharing, and communications for military application or for civilians through wireless sensor networks. But, in the best of our knowledge, none of the previous works have focused on the context of video games.

In this paper we evaluate the performance of four MANET broadcast schemes in the specific context of multiplayer video games support. Each scheme is representative of a particular approach. We analyze their ability to satisfy the requirement of multiplayer games in term of reliability, delays and energy consumption. We propose the assessment of different configurations depending on the game architecture, the network density and the parametrization of the broadcast schemes.

The remainder of this paper is organized as follow: in the next section, we detail the two common network game architectures, namely: C/S and P2P. Related works on MANET broadcasting schemes are reviewed in section 3. Section 4 gives a detailed description of our simulations scenarios. Obtained results of the assessments of MANET broadcast schemes in the application context of multiparty video gaming are exposed and analyzed in section 5. Finally, section 6 summarizes the main contributions of this work and provides some future prospects.

\section{GAME ENGINE ARCHITECTURES}

The game engine is a software providing all the needed functionalities to play video games using a hardware terminal (e.g. console or a PC). Typically, it includes several engines, where the most common ones are: $2 \mathrm{D} / 3 \mathrm{D}$ graphic render, physics engine, sound module, artificial intelligence engine, scene graph, animation module, threading, and networking system. The latter one is particularly important when the multiplayer mode is proposed by the game. Basically, the networking system is in charge of transmitting the game's data between all the entities involved in the game. The nature of these entities depends on the architecture of the game: Client/Server or Peer-to-Peer.

Actually, most of the multiplayer games rely on a centralized Client-Server (C/S) architecture. In this case, one part of the game software (the slave) is located in each client, and the second part (the master) in a central server, which coordinates the game. The clients are responsible of collecting and sending, to the server, all primitive commands related to the players' actions within the game, such as avatar control commands (movements, shooting, etc.). On the other hand, the server is responsible for collecting the game actions received from each client in order to compute the overall game state. This overall game state is then shared back (e.g. generally, using LAN broadcast messages), by the server, to all the clients. The $\mathrm{C} / \mathrm{S}$ architecture can provide good performances (consistent overall game state) under the condition of stable and low latency connections between the clients and the server. This is usually the case in wired LAN environment. However, when we consider a MANET environment, the $\mathrm{C} / \mathrm{S}$ architecture can raise many problems. Indeed, clients' mobility and channel condition can strongly affect the connectivity among terminals. Thus, the gameplay can strongly be degraded as the latency between the client and the server is increased. In fact, the gameplay is very sensitive to the round-trip-time (RTT) between the clients and the server. Smaller is the RTT, better is the gameplay.

An alternative to the $\mathrm{C} / \mathrm{S}$ architecture for multiplayer games is the Peer-to-Peer (P2P). In the latter architecture, there is no dedicated clients or server terminals, but only equal peer nodes that can simultaneously act as a client and a server. In this case, every peer can sends its local player actions to all other peers in a completely distributed manner, possibly, without the need of any central server. Thus, each peer is responsible of computing the overall game state from the received data. The main advantage of using this architecture resides in the inexistence of a central coordination point. Thus, P2P architecture seems more appropriated in MANET environment since it does not requires a persistent connectivity with a particular node (a server). However, it implies an efficient synchronization process between the peers.

In both $\mathrm{C} / \mathrm{S}$ and $\mathrm{P} 2 \mathrm{P}$ architectures, broadcast is a fundamental communication service in most of LAN multiplayer games. Indeed, in $\mathrm{C} / \mathrm{S}$ architecture, the server must regularly transmit, using broadcast messages, the overall game state (e.g. object positions and speed, hits, score etc.) to all the clients. Similarly, in P2P architecture, each peer can broadcast its game state view to all other participant peers, so that all nodes can converge toward a synchronized game state. In the next section, we give a short overview on main broadcast schemes in MANET environment. 


\section{BROADCAST SCHEMES FOR MANET}

Different broadcast schemes for MANET environment have been proposed in the literature [10] [5] [8] [9] [7]. Williams and Camp [11] categorize the broadcasting schemes into four families: simple flooding, probability based methods, area based methods and neighborhood based methods.

The first family, simple flooding, is considered as the default solution for packet broadcasting in ad hoc networks. In this scheme, each node will be required to rebroadcast to its neighbors each received packet, one time. The main advantage of using simple flooding is that it is the only way ensuring that each broadcasted packet will be received by all nodes in the network. However, when considering high density network, $\mathrm{Ni}$ and al. [10] show that simple flooding may lead to serious messages redundancy and to high contentions. This problem is referred as the broadcast storm problem.

The second family, probability based methods, includes two broadcasting schemes [10]: probability based and counter based. In the first scheme, probability based, each node is given a predefined packet re-broadcasting probability $(p)$. Precisely, whenever a node receives a broadcast packet, it will rebroadcast it with a probability $(p)$, or drop it with a probability $(1-p)$. Note, that if $p=1$ then this scheme leads to simple flooding. In the second scheme, counter based, when a node receives a broadcast message it chooses a random buffering delay. During this delay a counter $c$ is setup by the node in order to count the number of received same copies of that message (broadcasted by other neighbor nodes). After the expiration of the buffering delay, the node rebroadcasts the message only if $c$ is higher than a predefined threshold $\mathcal{C}$. Obviously, counter based scheme, is particularly performant in a high dense networks with low values of $\mathcal{C}$, as several nodes will not have to rebroadcast packets, reducing by this way redundant transmissions.

In the third family, referred as area based methods, packet's re-broadcasting decision is made upon the estimation of the relative physical distance between nodes. Within this family, we can cite two schemes [10]: distance based and location based. In the first scheme, each node is given a predefined distance threshold $(d)$. When a node receives a broadcast packet it estimates the physical distance separating it from the last relaying node, which sent that packet. The packet is dropped if the evaluated distance is less than $d$. Otherwise, the packet is re-broadcasted. The main problem of this scheme is due to the accuracy of the estimated distance. One possible solution is to derive the distance from the measured signal strength of the received packet. Unfortunately, this method is not satisfactory in realistic ad hoc networks. In location based scheme, each node adds its location information in the header of each sent packet. Thus, packet re-broadcasting is done only if the additional coverage area added by the receiver is greater than a given threshold. Unfortunately, this scheme makes the strong assumption that each node knows its location (e.g. using the global positioning system - GPS). For cost reason, it is clear that such requirement for a geo-localization system could not be reasonably fulfilled in the context of video gaming upon cheap portable consoles.

Finally, neighborhood based methods are based on the exploitation of the neighborhood nodes' information. The mains schemes belonging to this family are: sefl pruning [5], dominant pruning [5], scalable broadcasting [8], multipoint relay [9], Ad Hoc broadcast protocol [7] and Simplified Multicast Forwarding for MANET [6]. In sefl pruning, each node piggybacks into each re-broadcasted packets its adjacent nodes. Thus, nodes that received this packet can check if their own adjacent nodes are the same than those piggybacked in the received packet. If yes, the packet is dropped. Otherwise, it is re-broadcasted. While sefl pruning exploits only one hop node information, the remaining schemes: dominant pruning, scalable broadcasting, multipoint relay, Ad Hoc broadcast protocol and SMF for MANET require that all nodes have knowledge of their two-hop radius neighbors. Unfortunately, all of neighborhood based methods require extra transmission overhead, especially in dense MANET.

In this paper, we evaluate the performances of some of the mentioned ad hoc broadcast schemes in the application context of multiplayer video games. From each family, we choose the simplest mechanisms, since we believe that a reduced complexity is a fundamental criteria when considering implementation in portable consoles. Following these considerations, we choose to assess four broadcast mechanisms, namely: simple flooding, distant based, probability based and self pruning.

Note that these mechanisms have already been assessed, mainly through simulations. However, in the best of our knowledge, all the evaluation studies have been conducted out of the particular context of video gaming. Traffic models, mobility models and performance metrics were specific to other applications, such as video or audio streaming, file sharing, VoIP and communications for military applications or for civilians through wireless sensors networks. The specific traffic models characterizing multiplayer video games (like those of FPS [2]), the relationship between interacting nodes (which will depends on the architecture nature: Client/Server or P2P), the node mobility and the network density (which characterize playing places, like playground) were not considered in any other previous studies. In this paper, we integrate all these considerations. Details on our simulations scenarios are given in the next section.

\section{SIMULATIONS SCENARIOS}

In order to compare and evaluate the impact of simple flooding, probability based, distance based and self pruning mechanisms on the performance of video games over mobile ad hoc networks, we carry out a set of simulation tests. We have implemented and integrated the four mentioned broadcast schemes in Glomosim 2.0 [4] simulator.

In our simulations setup, we have considered that each node has a radio propagation range of 120 meters and a channel capacity set to $2 \mathrm{Mb} / \mathrm{s}$. To model a playing place, like a school playground, we have assumed a limited area characterized by a square with a side size equal to 300 meters. We have supposed four possible configuration scenarios, depending on the total number of nodes (players' consoles) in the area. Precisely, we have run simulations assuming successively 4, 8, 12 and 16 participants. We believe that these values are representative of typical group sizes that should be observed for MANET's LAN parties.

In regards with radio coverage $(120 \mathrm{~m})$ and the area surface $\left(300 \times 300 \mathrm{~m}^{2}\right)$ we were faced to the initial nodes' distribution problem. Indeed, when considering the small number of nodes scenario (e.g. 4 or 8 ) and assuming uniform random distribution for initial nodes' placement, sampling 
may leads to a disconnected graph topology (all nodes are not reachable). This scenario is not realistic in the general context of MANETs and particularly in the context of multiplayer games application. A realistic scenario would be to assume that before starting a party, gamers are distributed so that they form a connected graph. If their initial positions when switching on the radio interface indicate that all the players are not reachable, we think that a realistic behavior would be that some players will move so that the connectivity is obtained for the entire group

Following this assumption, all initial nodes' placements generated in our simulation executions are obtained with a connected graph. In other words, in the coverage area of each node, there is at least another node. Sampling that led to a disconnected graph is simply discarded.

For the same reasons explained before, the node mobility model that we implemented for our experiments relies on the hypothesis that all nodes movement is conditioned by the maintenance of the graph connectivity. Of course, in practice, such assumption may not hold. However, in this work we kept this assumption for simplicity reasons and clarity of our results and analysis. Reliability issues resulting from disconnections due to MANET's nodes movements are kept for future work.

Following this analysis, we have discarded some classical node mobility models (e.g. random way point). Rather, we have proposed an adapted gamers behavior mobility model. A basic assumption relies on the fact that players will be concentrated on the game and thus should not move frequently. Since we consider pedestrians, then a moving node should correspond to a slowly walking person. Typically, we took an average speed of a moving node which varies between 1 and 2 meters per second. In our model, the trajectory of a moving node is linear with a maximum distance set to 10 meters. Once this distance reached, the player will remain on place for a randomly chosen time. The average value of this pause time is about 10 seconds.

Two traffic models were implemented, each one is supposed to reflect the realistic behavior of transmitted data during a LAN multiplayer game. Each model is associated to the game architecture: $\mathrm{C} / \mathrm{S}$ and $\mathrm{P} 2 \mathrm{P}$. In the first case, we set one node (node 0) as the hosting server. Broadcast messages are only generated by this node. We supposed an FPS (First Person Shooter) game gender (e.g. Counter Strike or Quake 3). Following approximately the traffic model described in [2] for such games, we assumed that the server generates a Constant Bit Rate (CBR) traffic, where a packet size of 127 bytes is generated every $62 \mathrm{~ms}$. The remaining nodes act as clients, sending to the server unicast messages according to a CBR model. Each client generates unicast packet of 82 Bytes every $40 \mathrm{~ms}$. We assumed that unicast packets are relayed using AODV protocol. Multipoint Hello messages are sent every 10 seconds. Note that we choose CBR model, rather than Lognormal or shifted Weibull models described in [2] to speed up the simulation time and to simplify the analysis.

In $\mathrm{P} 2 \mathrm{P}$ case simulations, all nodes broadcast their local game information states. Since we did not found in the literature any traffic model for FPS LAN multiplayer games based on a P2P game engine, we have supposed that the generated traffic per node, should follow the client model in the $\mathrm{C} / \mathrm{S}$ architecture (since in a well designed $\mathrm{P} 2 \mathrm{P}$ system, only local game state information needs to be sent). That is,

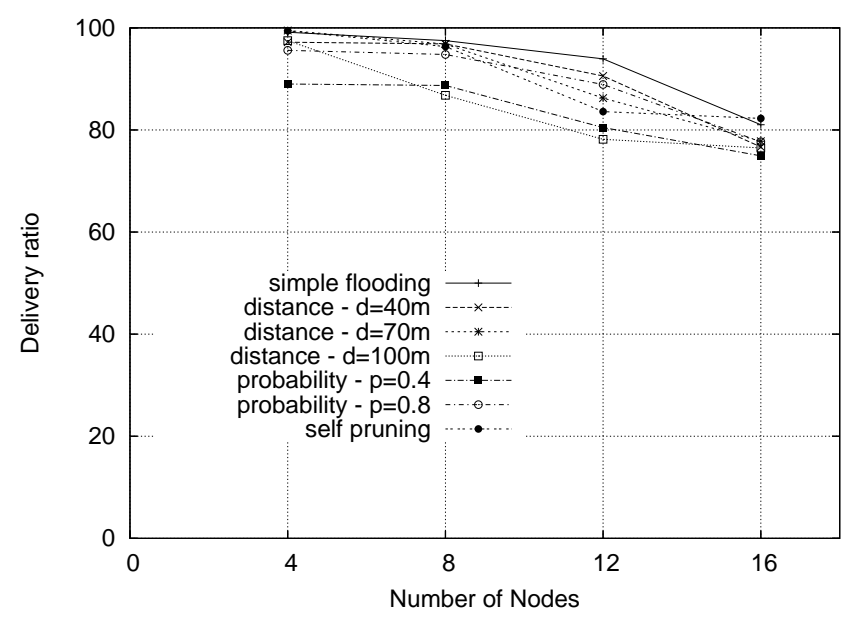

Figure 1: Packet delivery ratio for client-server architecture

each peer node generates a broadcast message of size equal to 82 bytes every $40 \mathrm{~ms}$.

In order to evaluate the ability of the probability-based scheme to adapt to the network density, we have considered two probabilities values: $p=0.8$ and $p=0.4$. The first value is expected to provide good results in a sparse network, while the second one should be more adapted for a dense network.

Similarly, we have considered three configurations distance values for the distance-based version. Precisely, we considered the following cases: $d=40, d=70$ and $d=100$ meters. The smallest value of $d$ is expected to provide the better results in the case of dense network.

Following all these assumptions, we have run extensive simulations for all possible scenarios described in this section. For each experiment, simulation duration times were set to 5 minutes. Results are exposed and analyzed in the next section.

\section{RESULTS AND ANALYSIS}

In figures 1 and 2, we show the average packet delivery ratio for respectively client-server and peer-to-peer architectures. We plot the packet delivery by considering different number of players (gaming consoles) (4, 8, 12 and 16 players). For $\mathrm{C} / \mathrm{S}$ architecture, the obtained results show that for all broadcasting schemes the packet delivery ratio is up to $75 \%$. The best performance is obtained by using simple flooding. We note also that performances are affected by the value of the re-broadcasting distance and the re-broadcasting probability. When we increase the rebroadcasting distance, we observe a decrease in the packet delivery ratio, and when we increase the re-broadcasting probability, we observe an increase in the packet delivery ratio. In $\mathrm{P} 2 \mathrm{P}$ architecture, the observation is different from $\mathrm{C} / \mathrm{S}$ architecture. Here, the best performance is obtained by using self pruning scheme, for 4 and 8 nodes. However, for 12 and 16 nodes the best performance is obtained when we used distance based scheme (for $d=100 \mathrm{~m}$ ). Furthermore, we notice that enhancing the number of players affects strongly the performances of $\mathrm{P} 2 \mathrm{P}$ architecture. This is mainly due to the huge number of transmitted packets, produced by the $\mathrm{P} 2 \mathrm{P}$ network. 


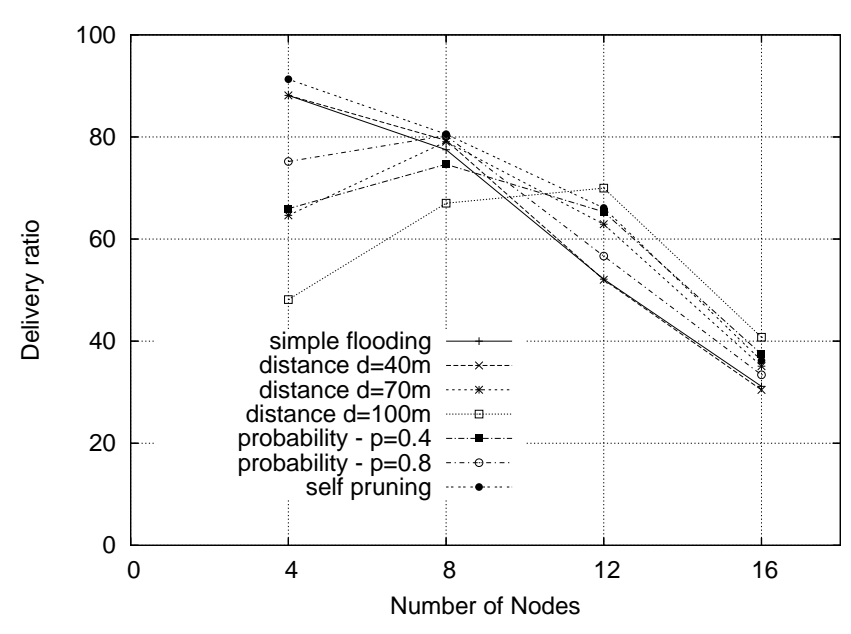

Figure 2: Packet delivery ratio for peer-to-peer architecture

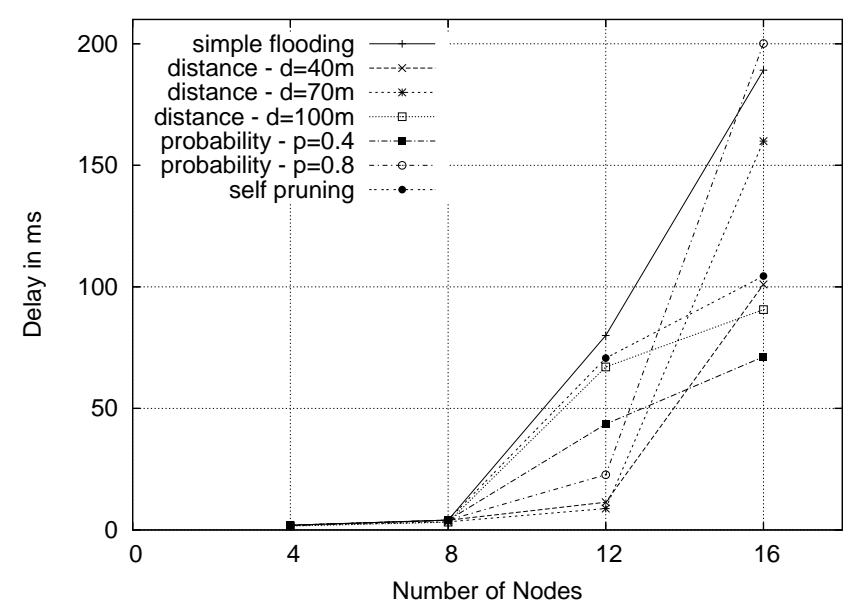

Figure 3: Packet delay for client-server architecture

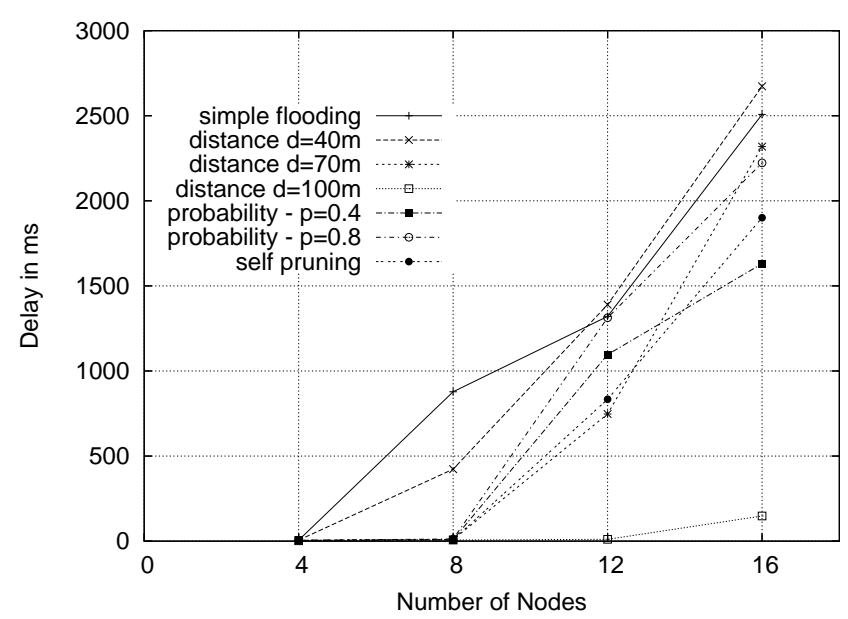

Figure 4: Packet delay for peer-to-peer architecture

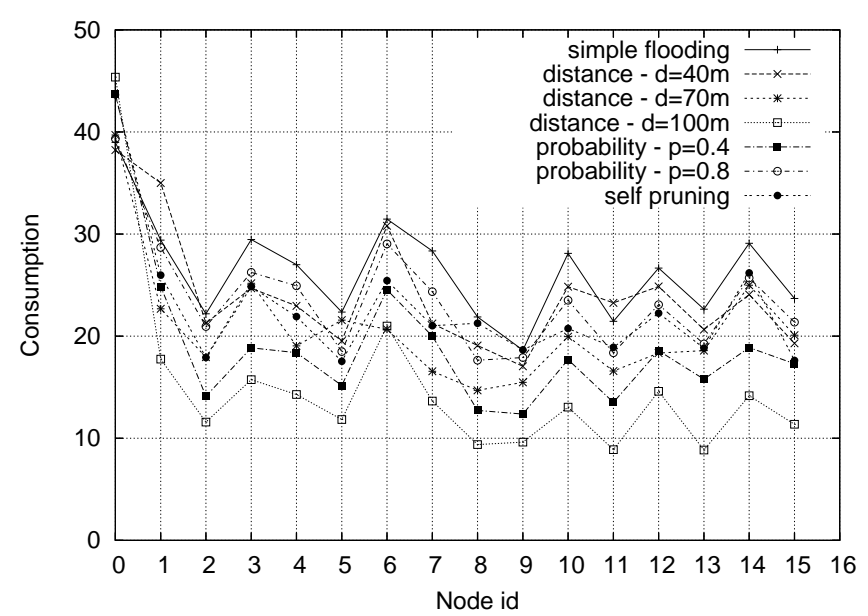

Figure 5: Energie consomption for client-server architecture

As for packet delivery ratio, the gameplay is strongly affected by the end-to-end delay. Lower is the end-to-end delay, better is the gameplay. In order to evaluate the impact of the broadcast algorithms on the end-to-end delay, we plot, in figures 3 and 4 , the average of maximum delay taken by each packet to reach the last destination node, for respectively $\mathrm{C} / \mathrm{S}$ and $\mathrm{P} 2 \mathrm{P}$ architectures. We mean by the last destination node, the last node in the network that received the message. In the case of $\mathrm{C} / \mathrm{S}$ architecture we consider only packets sent by the server, since we assume that the latter one is the only game terminal sending broadcast packets. In the case of $\mathrm{P} 2 \mathrm{P}$ architecture, we consider all packets sent by all terminal nodes. The obtained results show that, in $\mathrm{P} 2 \mathrm{P}$ architecture, except for distance based scheme (for $d=100 \mathrm{~m}$ ) the average end-to-end delay is very high (greater than $1.5 s$ for 16 players). However, the average end-to-end delay obtained for the $\mathrm{C} / \mathrm{S}$ architecture is acceptable(less than $200 \mathrm{~ms}$ for 16 players). The main problem of $\mathrm{P} 2 \mathrm{P}$ architecture is due to the huge number of re-broadcasted messages in the network, which increases highly the contention.

The battery life is one of the most serious issues for portable gaming consoles. Generally, default battery lifetime varies widely depending on application. For example, the PlayStation Portable's lifetime varies from less than 3 hours while accessing a wireless network and having screen brightness on its highest settings, to more than 10 hours during MP3 playback with the screen turned off. In order to evaluate the impact of broadcast algorithm on battery lifetime we plot, in figures 5 and 6 , the consumed energy for each node, for respectively $\mathrm{C} / \mathrm{S}$ and $\mathrm{P} 2 \mathrm{P}$ architectures. We consider here a network game of 16 players. In our simulations we measured the energy consumption at the radio layer. According to the specification of IEEE 802.11-compliant WaveLAN-II [3] from Lucent, the power consumption varies from 0.045 Watts in sleep mode to 1.25 and $1.50 \mathrm{Watts}$ in receiving and transmitting modes, respectively. The instantaneous power is multiplied by time delay to obtain the energy consumed. For example, data transmission of a 127-byte packet consumes $0.762 \times 10-3$ Joules $(1.50 \mathrm{~W}$ atts $\times 127 \times 8$ bits $/ 2000000 \mathrm{mbps})$. It is important to notice that in our simulation of the $\mathrm{C} / \mathrm{S}$ 


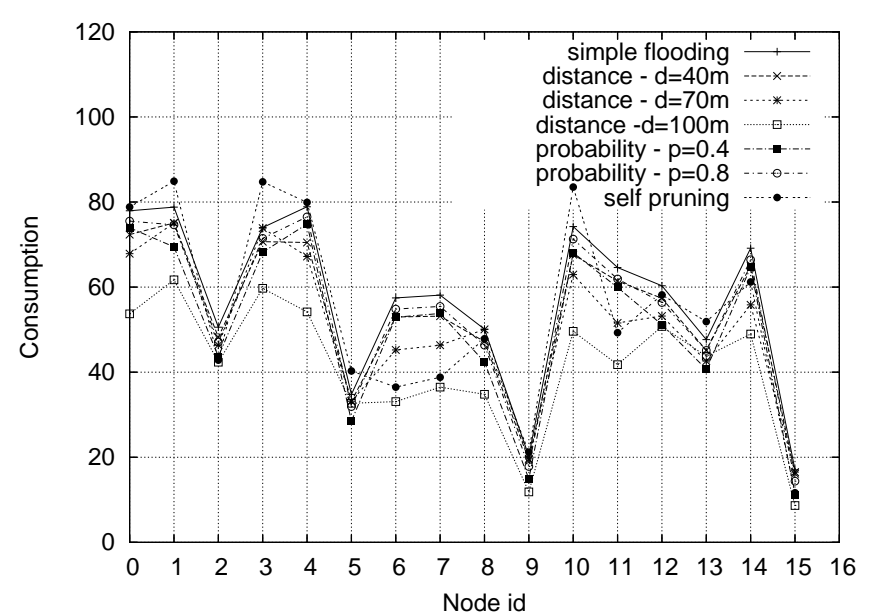

Figure 6: Energie consomption for peer-to-peer architecture

architecture, we add the impact of the packets transmission from the clients to the server. We can see clearly, from the two figures that the average energy consumed in P2P architecture is greater than the average energy consumed in the $\mathrm{C} / \mathrm{S}$ architecture. As mentioned above, this is due mainly to the number of packet re-broadcasted in $\mathrm{P} 2 \mathrm{P}$ architecture.

\section{CONCLUSION AND FUTURE WORKS}

Next generation of multiplayer games are expected to support MANET mode as mobile devices and wireless communication technologies are becoming ubiquitous. However, this is a challenging task and several issues still have to be addressed to make such scenario a reality in the near future. In this paper, we are dealing with MANET broadcast optimizations for multiplayer games since broadcast is a fundamental network service for such applications. We evaluated the performance of the following broadcast schemes: (distance-based, probability-based and self-pruning) for both Client/Server and P2P game architectures. Results show that broadcasting under the assumption of $\mathrm{C} / \mathrm{S}$ architecture is globally satisfying the gameplay requirements. In particular, for low distance-based and probability based parameters values. A drawback appears on the server's node energy consumption. This is a sensitive point since the server node will also have to consume more energy to compute global game state. One could reasonably expect that a better solution for load balancing the energy consumption relies on P2P architecture. However the results show a high degradation of delays and energy consumption even for P2P (due to the fact that more nodes are broadcasting). Exception made for distance based with a large distance value $(100 \mathrm{~m})$, but at the cost for less reliability. Of course, these "bad" results should be counter balanced by the fact that the CPU for computing the game state is balanced over all nodes (contrarily to the $\mathrm{C} / \mathrm{S}$ case, where it was concentrated on the server). Our experiments clearly show the need for designing an efficient and adapted broadcast MANET protocol that can scale with the constraints and specificities of the video game support. Our future work will concern effects of disconnections and the analysis of the network parameters that affect the quality of the gameplay. To that end, we will work on objective assessment of the gameplay.

\section{ACKNOWLEDMENTS}

This work was supported, in part, by the French National Research Agency (ANR) under the RIAM MADGAMES project.

\section{REFERENCES}

[1] Ietf manet wg (mobile ad hoc network). IETF, www.ietf.ora/html.charters/manet-charter.html.

[2] J. Färber. Network game traffic modelling. In NetGames '02: Proceedings of the 1st workshop on Network and system support for games, pages 53-57, New York, NY, USA, 2002. ACM Press.

[3] A. Kamerman and L. Monteban. Wavelan-11: A high-performance wireless lan for the unlicensed band. In Bell Labs Technical Journal, pages 118-133, Summer 1997.

[4] U. P. C. Laboratory and W. A. M. Laboratory. Glomosim: A scalable simulation environment for wireless and wired network systems. In Proceedings of the 3rd International Working Conference on Performance Modelling and Evaluation of Heterogeneous Networks (Het-Net'05), 2002.

[5] H. Lim and C. Kim. Multicast tree construction and flooding in wireless ad hoc networks. In MSWIM '00: Proceedings of the 3rd ACM international workshop on Modeling, analysis and simulation of wireless and mobile systems, pages 61-68, New York, NY, USA, 2000. ACM Press.

[6] J. Macker. Simplified multicast forwarding for manet. IETF Internet-Draft. March 2007.

[7] W. Peng and X. Lu. Ahbp: An efficient broadcast protocol for mobile ad hoc networks. J. Comput. Sci. Technol., 16(2):153-167, 2001.

[8] W. Peng and X.-C. Lu. On the reduction of broadcast redundancy in mobile ad hoc networks. In MobiHoc '00: Proceedings of the 1st ACM international symposium on Mobile ad hoc networking $\&$ computing, pages 129-130, Piscataway, NJ, USA, 2000. IEEE Press.

[9] A. Qayyum, L. Viennot, and A. Laouiti. Multipoint relaying: An efficient technique for flooding in mobile wireless networks. Technical Report Research Report RR-3898, INRIA, february 2000.

[10] Y.-C. Tseng, S.-Y. Ni, Y.-S. Chen, and J.-P. Sheu. The broadcast storm problem in a mobile ad hoc network. Wirel. Netw., 8(2/3):153-167, 2002.

[11] B. Williams and T. Camp. Comparison of broadcasting techniques for mobile ad hoc networks. In MobiHoc '02: Proceedings of the 3rd ACM international symposium on Mobile ad hoc networking E computing, pages 194-205, New York, NY, USA, 2002. ACM Press. 